\title{
La pseudociencia y el poder de los medios de comunicación. La problemática ausencia de bases teóricas para afrontar el fenómeno
}

\author{
Felipe Alonso MARcos \\ Universitat Pompeu Fabra \\ felipe.alonso@upf.edu \\ Sergi CoRTIÑAS Rovira \\ Universitat Pompeu Fabra \\ sergi.cortinas@upf.edu
}

\begin{abstract}
Resumen:
El presente artículo tiene la voluntad de señalar la urgente necesidad de realizar un esfuerzo teórico que permita afrontar los peligros derivados de la pseudociencia, peligros que aumentan si atendemos a su incorporación como contenido mediático. Desde una óptica transversal, al reflexionar desde varios ámbitos de estudio como son las teorías de la comunicación, la epistemología de la ciencia y la deontología periodística, los autores proponen una definición de pseudociencia y señalan diferentes líneas de actuación que consoliden y delimiten este objeto de estudio específico.
\end{abstract}

Palabras clave: pseudociencia; medios de comunicación; epistemología de la ciencia; divulgación de la ciencia; poder mediático

\section{Pseudoscience and the power of the media. The problematic lack of theoretical foundations to tackle the phenomenon}

\begin{abstract}
:
The present article aims to point out the urgent need of making an effort in order to face the threats of pseudoscience, threats that increase if we pay attention to its incorporation as media contents. From a transversal point of view, including communication theories, science epistemology and journalism ethics, the authors propose a new definition of pseudoscience and indicate different courses of action that can help to delimit and establish this specific object of study.
\end{abstract}

Keywords: pseudoscience; media; science epistemology; popularization of science; media power

\section{Referencia normalizada:}

Alonso Marcos, F. y Cortiñas Rovira, S. (2014): La pseudociencia y el poder de los medios de comunicación. La problemática ausencia de bases teóricas para afrontar el fenómeno. Historia y Comunicación Social. Vol. 19. Núm. Especial Marzo. Págs. 93-103.

Sumario: 1. Introducción. La problemática de la pseudociencia. 2. Aproximación a la pseudociencia. 3. El poder mediático. 4. Líneas de actuación propuestas a modo de conclusión. 5. Referencias. 


\section{Introducción. La problemática de la pseudociencia}

Uno de los más urgentes e importantes problemas a los que se ha de enfrentar la sociedad actual es la proliferación de la pseudociencia. El fenómeno, entendido de manera genérica como 'falsa ciencia', ha sabido introducirse en el tejido social y manejar las propias herramientas comunicativas del sistema. Esta inserción social comporta una serie de peligros frecuentemente infravalorados por la mayoría de la población. Entre ellos, podemos destacar que la pseudociencia (a) desvirtúa la ciencia al intentar usurpar su status y simular lo que no es; (b) causa un creciente embrutecimiento y retroceso cultural; (c) deriva, en la mayoría de ocasiones, en casos de fraude con un propósito deliberado de lucro; (d) manipula las emociones del gran público como pueden ser la esperanza, el miedo o la ingenuidad; y (e) es capaz de seducir con promesas vacías a algunos pacientes, en mayor o menor grado desesperados, para que abandonen las terapias médicas convencionales.

El hecho de que la pseudociencia haya conseguido acceder a la esfera de los medios de comunicación refleja una de las características principales de nuestra sociedad actual: su destacado componente paradójico. Autores como Bauman (2009), Beck (1998) o Hardt y Negri (2005) consideran que la complejidad, y la incertidumbre, el riesgo o la consecuente omnipresente sensación de crisis que de ella se derivan, son algunos de los conceptos clave para entender el espíritu de nuestra época.

Este componente paradójico de la sociedad actual puede verse reflejado en los paralelos caminos que recorren ciencia y pseudociencia. Si bien la ciencia ha llegado a metas y ha cumplido objetivos y expectativas nunca antes vistas o soñadas, la pseudociencia opone su propio crecimiento y desarrollo al auge científico contemporáneo. El nivel de profesionalización de algunas prácticas pseudocientíficas como la homeopatía proviene básicamente del nuevo dominio de las propias herramientas del sistema, principalmente las de mercado y las de comunicación. Este dominio del sistema en beneficio propio conduce a un alto nivel de inserción social, basado en la colaboración directa de grandes sectores sociales como son la publicidad, los medios de comunicación, las distribuidoras y las empresas privadas. La pseudociencia, por lo tanto, florece y se perfecciona al amparo de parte del sistema, lo que agrava sus potenciales amenazas y peligros. La inserción social de una determinada pseudociencia amplía su horizonte puesto que presenta dicha pseudociencia como un elemento social más para el cual se ha provocado un clima de normalidad e inocuidad nada beneficioso. Los medios de comunicación, como entes estructurantes de la sociedad en la que vivimos, tienen la capacidad de normalizar conductas y opiniones, y esa capacidad de imponer narrativas sobre la realidad facilita la interiorización por parte del público de una nociva sensación de familiaridad ante los fenómenos pseudocientíficos.

Para minimizar los riesgos de la pseudociencia la herramienta más potente es la divulgación. La divulgación científica tiene como objetivo principal rebajar la brecha intelectual entre conocimiento especializado y conocimiento popular. Gran parte de esta responsabilidad divulgativa relativa a la ciencia recae en los hombros de aquellos 
profesionales del periodismo científico y de los propios científicos, en cuyas tareas se incluye el esfuerzo de acercar de forma clara y comprensible su conocimiento a la figura del gran público. En el caso que nos ocupa la divulgación de la ciencia ha de proporcionar unas herramientas de crítica y un modelo definido de qué es ciencia y qué no lo es a modo de criterio que pueda contrarrestar el avance y la inserción de la pseudociencia.

Paradójicamente, los medios de comunicación son a su vez los principales aliados de la ciencia en su afán divulgativo y sus principales enemigos al dar cobijo en sus contenidos a fenómenos pseudocientíficos. Este comportamiento dual, incoherente viola los principales códigos deontológicos y las regulaciones éticas. Debido al gran poder de los medios de comunicación como difusores de realidad y avaladores de aquello incluido / excluido en el sistema, su responsabilidad no es un tema menor, ya que puede multiplicar exponencialmente las amenazas de la pseudociencia, a la vez que justifica la realización de un esfuerzo teórico para afrontar el problema.

\section{Aproximación a la pseudociencia}

El estudio clásico de la pseudociencia se relaciona con la epistemología y la filosofía de la ciencia, ya que forma parte del asunto de la demarcación de la ciencia (p.e. Bunge, 1972; Khun, 2006; Lakatos; 1993; Popper, 2011; Russell, 1975). Lakatos (1993:16) señala al respecto que "la demarcación entre ciencia y pseudociencia no es un mero problema para filósofos de salón; tiene una importancia social y política vital".

De tal afirmación se sigue que el principal obstáculo a la hora de combatir la pseudociencia es la incomprensión, tanto a nivel global como específico, del fenómeno. La pseudociencia funciona como una construcción teórica que, a modo de paraguas conceptual, engloba una gran serie de prácticas, disciplinas, productos, fenómenos y poderes. Su carácter caleidoscópico y difuso, a la vez que dificulta su sistematización desde un ámbito de estudio, facilita su permanencia y expansión en el tejido social.

A la pseudociencia se le atribuyen como características principales el hecho de establecer relaciones causales acerca de fenómenos que la ciencia considera imposibles (Sokal, 2006); el hecho de no utilizar métodos rigurosos en sus investigaciones, carecer de armazones conceptuales contrastables y afirmar resultados positivos sin pruebas cuestionables mediante investigadores parciales (Kurtz (1978/81); o por promover enunciados factuales no garantizados epistemológicamente (Fuller, 1985). Otros autores como Lilienfeld et al, (2003) añaden que la pseudociencia no se define por su objeto de estudio, sino por su manera de aproximarse a los resultados. Hanson (2009, Pág.240) atribuye una triple característica a la pseudociencia: la pertenencia a una disciplina que tiene relación (de una manera muy amplia) con la ciencia; (2) que no está epistemológicamente garantizada; y (3) que sus defensores tratan de crear la impresión de que es científica. 
La aproximación de la escuela norteamericana, compuesta por autores como Schermer (1997), Dawkins (1998), Gardner (1988), Randi (1982) o Sagan (2000), se ha basado en resaltar el carácter irracional, espectacular y fraudulento del fenómeno y no tanto en la óptica epistemológica.

Según Sagan, el poder de la pseudociencia es su capacidad para ofrecer asideros emocionales que mitigan miedos inherentes al ser humano.

[La pseudociencia] colma necesidades emocionales poderosas que la ciencia suele dejar insatisfechas. Proporciona fantasías sobre poderes personales que nos faltan y anhelamos [...]. En algunas de sus manifestaciones ofrece una satisfacción del hambre espiritual, la curación de las enfermedades, la promesa de que la muerte no es el fin (Sagan, 2000. Pág. 24-25).

Para Shermer la razón es similar, ya que la pseudociencia seduce con falsas promesas a aquellos individuos que pasan por momentos difíciles.

La vida es contingente y llena de inseguridades, siendo la que más nos asusta el modo, el momento y el lugar de nuestra propia defunción. [...] Bajo la presión de la realidad, nos volvemos crédulos. Buscamos certezas reconfortantes en los videntes y los charlatanes, los astrólogos y los psíquicos. Nuestras facultades críticas colapsan ante la avalancha de promesas y esperanzas ofrecidas para aliviar las mayores cargas de la vida (Shermer, 1997. Pág. 5).

Estos autores se centran en la vertiente psicológica del fenómeno, a la vez que mantienen una postura de militancia activa con el fin de desenmascarar las diferentes manifestaciones de la pseudociencia. Esta escuela de pensamiento sostiene que la mejor opción es ridiculizar la pseudociencia, puesto que argumentar racionalmente sus limitaciones no consigue derrotar a sus partidarios. Gardner señala que si solamente se combate mediante la divulgación científica se trata de una lucha indirecta, y nunca lo suficientemente efectiva. La fórmula 'a más divulgación, menos pseudociencia' no funciona. Según Gardner, sus partidarios no atienden a argumentos racionales en contra puesto que son una minoría previamente convencida e impermeable a la divulgación.

A la hora de discutir extremos en materia de heterodoxia en ciencia, considero una pérdida de tiempo ofrecer argumentos racionales. Aquellos que están de acuerdo no necesitan recibir educación en lo que se refiere a cuestiones tan triviales, e intentar convencer a los que no están de acuerdo es como intentar escribir sobre agua (Gardner, 1988. Pág. 14).

Más allá de la divulgación básica, es necesaria una casuística que detecte, una por una, las manifestaciones pseudocientíficas, para a continuación exponer el por qué sus pretensiones carecen de valor, no pueden ser consideradas científicas y alertar de qué peligros encierran. 


\section{El poder mediático}

Desde las teorías de la comunicación encontramos una serie de autores (McQuail, 2000; Noelle-Neumann, 1973, 1995; Marletti, 1985; Lippmann, 2003, entre otros) y teorías (espiral del silencio, agenda-setting, tematización, framing) que han establecido un paradigma sobre los efectos de los medios de comunicación basado en la visión de éstos como entes estructurales de la realidad. Los medios mantienen un acceso democrático a la cultura y la información, construyen la realidad social mediante sus las narraciones y discursos, sostienen las dinámicas de creación y fluctuación de la opinión pública, generan consenso y/o señalan las conductas y opiniones etiquetadas como desviadas, y, en su visión más extrema, se configuran como una herramienta de control biopolítico.

Según este modelo, los medios de comunicación ejercen un poder notable capaz de generar efectos sobre los individuos y/o la sociedad a largo plazo, de manera tanto voluntaria como involuntaria, que afectan el modo de entender y relacionarse con la realidad. Estos efectos se centran en la construcción común de significado y en cómo la audiencia incorporan o no los mensajes, mediante un proceso de negociación, en sus estructuras personales previas, configuradas a su vez por anteriores identificaciones colectivas (McQuail, 2000. Pág.499).

Si bien este paradigma se desmarca de los efectos todopoderosos del modelo hipodérmico, devuelve parte de ese poder a los medios de comunicación que había sido negado en el modelo de los efectos mínimos originado bajo el amparo de la investigación administrada norteamericana (Klapper, 1957, 1974). Los resultados empíricos que sostuvieron el modelo de los efectos mínimos fueron concebidos bajo la tutela de una serie de investigaciones subvencionadas por empresas, medios de comunicación y ejército, quienes querían un modelo que les librara de la responsabilidad que conlleva un gran poder mediático. Como apunta Rodrigo (2001. Pág.18), "la perspectiva conductista [propia del modelo hipodérmico] no era bien aceptada por la ideología liberal capitalista norteamericana porque mostraba una imagen de los medios de comunicación que podía provocar el intervencionismo gubernamental". Unos medios de comunicación sin poder evitaban una regulación estatal; los individuos, por tanto, son capaces de equilibrar e incluso sobreponerse al poder mediático. Los medios, bajo esta óptica, tan sólo cubren las necesidades de los individuos y se someten a las leyes de mercado basadas en la oferta y la demanda.

Es a partir de los años setenta del pasado siglo cuando se produjo un retorno a la concepción de los medios de comunicación como productores de efectos poderosos, y por tanto, cuando se produjo un retorno a las preocupaciones deontólogicas, éticas e ideológicas. Este nuevo modelo, que recupera una visión poderosa de los medios de comunicación, no es en conjunto la negación de los elementos sociológicos y psicológicos que contribuyeron a formar el modelo de los efectos nulos, ni siquiera una visión de los medios de comunicación como omnipotentes y todopoderosos que si mantuvo su vigencia en la teoría crítica europea personificada en la Escuela de Frankfurt, sino una especie de mezcla de efectos poderosos bajo condiciones limi- 
tadas (Igartua y Humanes, 2004). Es el individuo quien limita el gran poder de los medios de comunicación, en una constante negociación de consumo seleccionado hasta el límite por parte del sujeto y una omnipresente oferta de comunicación mediática acumulativa.

La visión más radical de este poder estructural supone la creación mediática del pseudoentorno (Lippmann, 2003) o realidad alternativa que suplanta el mundo real como resultado, no tanto de la manipulación, sino de la reducción económica de los esfuerzos por hacer de la realidad algo manejable y explicable. Según este planteamiento, recogido por autores como Baudrillard (2008) o Boorstin (2012), el ecosistema mediático se nutre básicamente del pseudoevento (Boorstin, 2012), o cae en el exceso del simulacro (Baudrillard, 2008). Mientras que el pseudoevento hace referencia al conjunto de situaciones puntuales creadas exclusivamente para su consumo mediático, es decir, a situaciones que no existirían sin el apoyo mediático que brindan los medios, en el simulacro se trata de la suplantación global de la realidad por eventos que simulan la propia realidad. La era postsocial de la información y es la era de simulación, donde para ocultar el vacío social de nuestra sociedad y su ausencia de sentido, se crean y recrean numerosos pseudoeventos que tan sólo aportan sensación de continuidad y seguridad al individuo, que cree vivir en una sociedad coherente y con una dirección y sentido.

Una serie de autores contemporáneos como Deleuze y Guattari (2010a, 2010b), Raunig (2008) o Lazzarato (2008), pese a dedicarse a la filosofía o la sociología, han teorizado de manera amplia y profusa sobre el papel social de los medios de comunicación. Van un paso más allá del modelo basado en los efectos poderosos bajo condiciones limitadas y agravan el poder de los medios de comunicación al considerarlos como dispositivos de control capaces de normativizar la realidad y subjetivizar al individuo. Es a partir principalmente de la lectura que hacen estos autores del concepto de 'biopoder' foucaltiano y la trasformación de la sociedad postfordista al actual sistema tardocapitalista, que podemos hablar de dos grandes bloques de efectos: la servidumbre maquínica (asservissement machinique) y la sujeción social (assujettissement sociale).

Hemos de tomar a los medios de comunicación como una máquina técnica, y ésta no ha de ser pensada únicamente a partir de sus componentes y funcionamiento, sino como una máquina total que despliega comportamientos y pautas sociales; una máquina, que, independientemente de sus procesos y sus elementos, se configura a sí misma a través del uso que le de el sujeto. Por lo tanto, desarrolla sus características a la vez que se establece como parte orgánica del cuerpo social y adquiere una plusvalía a causa de su potencial para modificar las costumbres de los individuos.

La máquina social que forman individuos y medios de comunicación, es decir, el acoplamiento entre individuos y medios, permite a éstos últimos la producción, no sólo de noticias, entretenimiento u otros contenidos, sino también de la subjetivación normativa que comporta la inscripción del sujeto en una sociedad determinada. Para Lazzarato, la sujeción social es la inscripción del sujeto en el cuerpo social que 
permite la interiorización por parte del sujeto de un poder sutil basado en la autocensura.

La sujeción social actúa sobre la dimensión molar del individuo (su dimensión social, sus roles, sus funciones, sus representaciones), mientras que la servidumbre maquínica actúa sobre la dimensión molecular, preindividual, infrasocial (los afectos, las sensaciones, los deseos, las relaciones aun no individualizadas, no asignables a un sujeto) (Lazzarato, 2008. Pág. 110).

Aceptemos o no esta visión radical de los medios de comunicación como herramienta extensiva del poder, hay que reconocer su innegable capacidad para generar efectos poderosos a largo plazo en la sociedad y entre los individuos. Las amenazas de la pseudociencia, por tanto, se multiplican si ésta es capaz de acceder y encontrar en los medios de comunicación una plataforma que le facilite la inserción social y legitime su discurso al amparo del metadiscurso mediático.

\section{Líneas de actuación propuestas a modo de conclusión}

La investigación sobre las sinergias establecidas entre periodismo y pseudociencia es un ámbito de estudio extremadamente importante debido a que las amenazas de la pseudociencia se multiplican a partir de su exposición mediática. Los estudios etnográficos del periodismo y sobre medios de comunicación deben reaccionar ante los peligros de la pseudociencia y analizarla desde diferentes ángulos como pueden ser: (1) índices de presencia; (2) tratamiento y cobertura; (3) vínculos entre publicidad y pseudociencia; (4) creencias personales de los periodistas; (5) intereses de terceros; (6) formación y experiencia del periodista; (7) existencia, aplicación y/o creación de códigos deontológicos específicos; y (8) percepción y conocimientos de la figura del editor al respecto. Sin embargo, las investigaciones que hasta ahora se han dedicado a analizar dichas relaciones lo han hecho de una manera superficial y además ocupan un lugar marginal en los estudios sobre comunicación.

Pese a que la investigación previa sobre ética periodística representa un ámbito de estudio consolidado en la comunidad académica internacional, y también de manera específica en España (Aznar, 2005; Camps, 2001; Casasús, 1997, 2001), tampoco ha tratado de forma concreta el fenómeno pseudocientífico. Según Ward (2008. Pág.139) la ética es "el análisis de la conducta correcta, la práctica responsable y las interacciones humanas justas a partir de los mejores recursos disponibles". Ya que el periodismo es básicamente el ejercicio de una profesión, la deontología periodística debe ser, en palabras de Ward (2008. Pág.139) un "tipo de ética aplicada", que básicamente investigue "los 'micro' problemas acerca de qué deben hacer los periodistas en determinadas situaciones, y los 'macro' problemas acerca de qué deberían los medios de comunicación, dado su rol en la sociedad". Alsius $(1998,1999,2011)$ ha detallado exhaustivamente el conjunto de cuestiones éticas del ámbito de la comunicación y las 
agrupa en torno a cuatro principios fundamentales como son (1) verdad; (2) justicia; (3) libertad; y (4) solidaridad o responsabilidad social.

El propio Alsius (2001. Pág. 51) reconoce que en su trabajo pueden haber quedado vacíos sobre materias de las cuales aun no se ha elaborado suficiente doctrina deontológica, o de materias que no han sido consideradas aun lo suficientemente importantes para figurar en él. Los presentes autores proponen la inclusión de un apartado específico para la pseudociencia, situado como un subgrupo más del punto 4.3. titulado 'materias de especial sensibilidad social', junto con los demás subgrupos que lo integran (1-incitación a la violencia; 2- información de mal gusto; 3-respeto a las creencias; 4- alarmismo; y 5- violencia de género). Estos subgrupos se sitúan bajo el principio general ético de responsabilidad social, el cual se refiere a una doble responsabilidad como parte del compromiso social: la institucional de los medios de comunicación y la individual del periodista.

Los autores del presente artículo proponen abordar las siguientes líneas de actuación para paliar la ausencia de un corpus teórico adecuado a partir del cual analizar de manera adecuada la pseudociencia y sus relaciones con los medios de comunicación: (1) un estudio epistemológico centrado en la definición del concepto y el criterio de demarcación científico; (2) una tipificación de las manifestaciones pseudocientíficas que permitan identificarlas una por una en una taxonomía; (3) la divulgación y discusión de términos conceptuales sobre los límites de la ciencia que permiten obtener una visión global de la problemática; y (4) la elaboración de unos criterios éticos que fundamenten una deontología específica para guiar el tratamiento mediático de la pseudociencia.

Como primer resultado, y tras un estudio de la literatura clásica y académica respecto a la epistemología de la ciencia y su criterio de demarcación (Bunge, 1972; García, 2008; Hempel, 1976; Lakatos, 1993; Merton, 1977; Popper, 2011; Russell, 1975; Thagard, 1978), desde el presente artículo se esboza una definición de la pseudociencia centrada su vertiente comunicativa. A partir de las características que se atribuyen de manera consensuada a la ciencia podemos definir negativamente la pseudociencia como aquello que no cumple los requisitos científicos, a la vez que nos centramos en aquello que sí comparten, la posibilidad de ser comunicada. Es precisamente en su comunicabilidad donde radican sus peligros y donde nos sitúa paralelamente nuestro interés y su único rasgo positivo: la relación pseudociencia-medios de comunicación.

Por lo tanto, y de la manera descrita, proponemos definir la pseudociencia como: (1) el conjunto de prácticas que intentan apoderarse del status y el método científico, (2) mediante la exposición de resultados aislados, no demostrables, y/o puramente subjetivos, (3) obtenidos de manera acrítica, no sistemática, y/o interesada, (4) para generar un conocimiento no acumulable ni válido científicamente, (5) que opera en ámbitos donde la ciencia no llega o donde no ha ofrecido resultados satisfactorios, $\mathrm{y}$ (6) que suele ser hábil en el manejo de los medios de comunicación y de las emociones del gran público. 
Queremos remarcar el hecho, a pesar del riesgo de ser repetitivos, de que tras una comparación de las características de la ciencia y su cumplimiento e incumplimiento por parte de la pseudociencia, la única característica compartida por ambas es su naturaleza comunicable. Ahí radica la principal habilidad de la pseudociencia, y el núcleo a desactivar que genera todas sus amenazas, puesto que es capaz de extender su propagación en cualquier área de la sociedad, deseosa de darse a conocer mientras que pone en peligro el status científico al intentar usurparlo. Para conseguirlo, se hace hueco en los medios de comunicación, sobre todo en aquellas plataformas que anteponen el sensacionalismo y el espectáculo al rigor informativo, o sus intereses económicos a su responsabilidad para con la sociedad.

\section{Bibliografía}

ALSIUS, S. (1998): Ètica i periodisme. Barcelona: Pòrtic.

ALSIUS, S. (1999): Codis ètics del periodisme televisiu. Barcelona: Pòrtic.

ALSIUS, S. (2011): Cap a una gran base de dades per a l' estudi de l' ética periodística. Periodística, 13, 27-57.

AZNAR, H. (2005): Ética de la comunicación y nuevos retos sociales. Códigos y recomendaciones para los medios. Barcelona: Paidós.

BAUMANN, Z. (2009): Tiempos Líquidos. Viviendo en una época de incertidumbre. Barcelona: Tusquets.

BAUDRILLARD, J. (2008): Cultura y simulacro. Barcelona: Kairós.

BECK, U. (1998): La sociedad del riesgo. Barcelona: Paidós.

BOORSTIN, D. (2012): The Image: A Guide to Pseudo-Events in America. New York: Random House.

BUNGE, M. (1972): La ciencia, su método y su filosofía. Buenos Aires: Siglo XX.

CAMPS, V. (2001): El què i el com de la informació de qualitat. Quaderns del Consell de l'Audiovisual de Catalunya. No. 9 (pp. 12-18). Barcelona: Consell de l'Audiovisual de Catalunya.

CASASÚS, J.M. (2001): Perspectiva ética del periodismo electrónico. Estudios sobre el mensaje periodístico, 7, 49-55.

CASASÚS, J.M. (1997): Periodismo y comunicación ética. Bioètica \& debat: Tribuna abierta del Institut Borja de Bioètica, 10, 1-5.

DAWKINS, R. (1998): Unweaving the Rainbow. Boston: Houghton Mifflin.

DELEUZE, G. y GUATTARI, F. (2010a): El AntiEdipo. Capitalismo y esquizofrenia. Barcelona: Paidós.

DELEUZE, G. y GUATTARI, F. (2010b): Mil Mesetas. Capitalismo y esquizofrenia. Valencia: Pre-textos.

FULLER, S. (1985): The demarcation of science: a problem whose demise has been greatly exaggerated. Pacific Philosophical Quarterly 66, 329-41.

GARCÍA, L. (2008): Aproximación epistemológica al concepto de ciencia: una propuesta básica a partir de Kuhn, Popper, Lakatos y Feyerabend. Andamios, 8 (4), 185-212. 
GARDNER, M. (1988): La ciencia. Lo bueno, lo malo y lo falso. Madrid: Alianza.

HANSSON, S. (2009): Cutting the Gordian Knot of Demarcation, International Studies in the Philosophy of Science 23 (3), 237-43.

HARDT, M., y NEGRI, T. (2005): Imperio. Barcelona: Paidós.

HEMPEL, C. (1976): Filosofia de la ciencia natural. Madrid: Alianza.

IGARTUA, J.J. y HUMANES, M.L. (2004): Teoría e investigación en comunicación social. Madrid: Síntesis.

KLAPPER, J. (1957): What we know about the effects of mass communication: the brink of hope. The public opinion quarterly, 21 (4), 453-474.

KLAPPER, J. (1974): Efectos de las comunicaciones de masas. Madrid: Aguilar.

KUHN, T. (2006): La estructura de las revoluciones científicas. Madrid: Fondo de cultura económica de España.

KURTZ, P. (1978/1981). Is parapsychology a science? The Skeptical Inquirer, 2 (3), 14-23.

LAKATOS, I. (1993): La metodología de los programas de investigación científica. Madrid: Alianza.

LAZZARATO, M. (2008): Postfacio, en RAUNIG, Gerald, Mil Máquinas (Pp. 109-118). Barcelona: Traficantes de sueños.

LILIENFELD, S., LYNN, S., y LOHR, J. (2003): Science and pseudoscience in clinical psychology: Initial thoughts, reflections, and considerations. En LILIENFELD, Scott; LYNN, Steven y LOHR, Jeffrey (Eds.), Science and pseudoscience in clinical psychology (pp. 1-14). New York: Guilford.

LIPMANN, W. (2003): La opinión pública. Madrid: Langre.

MARLETTI, C. (1985): Prima e dopo. Tematizzacione e comunicazione política. Torino: RAI/ERI.

MERTON, R. (1977): La sociología de la ciencia. Madrid: Alianza.

MCQUAIL, D. (2000): Introducción a la teoría de la comunicación de masas. Barcelona: Paidós.

NOELLE-NEUMANN, E. (1973): Return to the concept of powerful mass media. Studies of broadcasting, 9, 67-112.

NOELLE-NEUMANN, E. (1995): La espiral del silencio. Opinión pública: nuestra piel social. Barcelona: Paidós.

RANDI, J. (1982): Flim-Flam! Psychics, ESP, Unicorns, and Other Delusions. New York: Prometheus Books.

RAUNIG, G. (2008): Mil Máquinas. Barcelona: Traficantes de sueños.

RODRIGO, M. (2001): Teorías de la comunicación. Ámbitos, métodos y perspectivas. Barcelona: Aldea Global.

RUSSELL, B. (1975): La perspectiva cientifica. Barcelona: Ariel.

SAGAN, C. (2000): El mundo y sus demonios. Barcelona: Planeta.

SHERMER, M. (1997): Why people believe in weird things. Pseudoscience, superstition, and other confusions of our time. New York: Freeman and Company.

SOKAL, A. (2006): Pseudoscience and Postmodernism: Antagonists or Fellow Travelers? En FAGAN, Garrett (Ed.) Archaeological Fantasies: How Pseudoarchae- 
ology Misrepresents the Past and Misleads the Public (pp. 286-361). New York: Routledge.

POPPER, K. (2011): Realismo y el objetivo de la ciencia. Post-scriptum a la lógica de la investigación cientifica, Volumen I. Madrid: Tecnos.

THAGARD, P. (1978): Why astrology is a pseudoscience. En ASQUITH, Peter y HACKING, Ian (Eds) PSA 1978: Proceedings of the 1978 Biennial Meeting of the Philosophy of Science Association, 1, 223-234.

WARD, S. (2008): Global Journalism Ethics: Widening the Conceptual Base. Global Media Journal -- Canadian Edition 1, 1, 137-149.

\section{Notas}

Este trabajo pertenece al proyecto de investigación "EL PERIODISMO CIENTIFICO EN ESPAÑA Y LAS NUEVAS TECNOLOGIAS DE LA INFORMACION (TIC): MAPA DE SITUACION Y PROPUESTAS DE ACTUACION PARA MEJORAR LOS PROCESOS COMUNICATIVOS" (Referencia: CSO2011-25969, Plan Nacional I+D+i convocatoria 2011), financiado por el Ministerio de Ciencia e Innovación, cuyo investigador principal es Sergi Cortiñas Rovira.

\section{Los autores}

Felipe Alonso es un investigador contratado del Departamento de Comunicación de la Universitat Pompeu Fabra (UPF). Es profesor en la Escuela de Publicidad y Relaciones Públicas (adscrita a la UB). Es miembro del Grup de Recerca en Comunicació Científica (GRECC). Sus líneas de especialización las teorías de comunicación, las teorías de los efectos, el periodismo científico y la pseudociencia en los medios.

Sergi Cortiñas es profesor permanente del Departamento de Comunicación de la Universitat Pompeu Fabra (UPF). Miembro del Grup de Recerca en Comunicació Científica (GRECC). Investigador Principal del proyecto "El periodismo científico en España y las nuevas tecnologías de la información (TIC): mapa de situación y propuesta de actuación para mejorar los procesos comunicativos" (CSO2011-25969). 